Les Bibliotheques DES EGLISES REFORMEES FRANÇAISES AU XVII ${ }^{\mathrm{B}}$ SIECLE:

le cas de la bibliotheque de l'Eglise de Paris (Charenton)

\title{
Marianne Carbonnier-Burkard
}

Dans le royaume de France pacifié par l'édit de Nantes, la minorité réformée a transposé pour son compte, à son échelle, le programme des villes et Etats protestants associant aux nouvelles Eglises des écoles et des bibliothèques. Dès 1596, les synodes des Eglises réformées exhortent les Eglises - du moins celles qui en ont les moyens, à «dresser des bibliothèques publiques pour servir aux ministres et proposans», et de même les Académies créées pour la formation des futurs pasteurs Nîmes, Montauban, puis Die, Sedan, Saumur. On ne sait presque rien des bibliothèques qui ont pu être ainsi dressées par des Eglises locales («bibliothèques consistoriales») et par les Académies². Laissons de côté les bibliothèques d'académie, indissociables de l'institution académique du monde réformé, modelée par Lausanne et Genève ${ }^{3}$. Restent les bibliothèques des Eglises locales. Combien des quelques 700 Eglises du royaume ont entretenu une bibliothèque? Moins d'une dizaine seulement sont bien attestées, à différentes dates: La Rochelle, Rouen, Bordeaux, Charenton, Beaune, Baugé-Angers, Issoudun ${ }^{4}$. De toute façon, aucune

1 Synode National de Saumur 1596, in: Jean Aymon, Tous les synodes..., La Haye, 1710, I, p. 203. Cf. Synode national de Jargeau 1601 (id., I, p. 237); Gap 1603 (id.,I, p. 276); La Rochelle 1607 (id., I, p. 313); Castres 1626 (id., II, p. 378).

2 Aperçu dans Louis Desgraves, «Note sur les bibliothèques protestantes», in: Claude Jolly, dir., Histoire des bibliothèques françaises, II, Paris, Promodis-Ed. du Cercle de la Librairie, 1988, p. 188-191.

3 Recherches en cours sur la bibliothèque de l'académie de Saumur: Thomas Guillemin, «Ex bibliotheca. Les livres retrouvés de l'académie protestante de Saumur», Exposition au Château-Musée de Saumur, 2019 (catalogue à paraître).

4 Références bibliographiques dans l'article de Louis Desgraves, cité ci-dessus note 2. A compléter par: Margreet Dielemann, Bibliothèques protestantes et catholiques à Baugé 1677-1685, Mémoire de Master 2, Angers, 2012. 
n'a survécu aux mesures de destruction et dispersion préalables à la Révocation de l'édit de Nantes, en 1685.

La bibliothèque de l'Eglise réformée de Paris, à Charenton, n'a pas échappé à ce sort, mais relevant de l'Eglise de la capitale elle a fait l'objet d'une sollicitude spéciale, tant des réformés que du pouvoir royal, même post mortem. Elle a ainsi laissé plus de traces que les autres, en particulier trois catalogues: un catalogue manuscrit commencé en 1626, inachevé, conservé au département des Manuscrits de la BnF, publié dans le BSHPF par Jacques Pannier, en 19065; et deux catalogues imprimés de vente aux enchères à La Haye, l'un de 1722, conservé à la BnF; l'autre de 1695, découvert récemment dans Books Sales Catalogues Online-Book Auctioning in the Dutch Republic, ca 1500-1800 (Leiden et Boston, Brill, 2015).

On présentera ici l'histoire de cette bibliothèque, passée de Charenton à La Haye, avec les éléments nouveaux tirés des catalogues de vente. On tentera ensuite de reconstituer le fonds de livres, en partant du catalogue manuscrit de Paris, puis en considérant les deux catalogues de vente de La Haye.

\section{L'HISTOIRE}

$\mathrm{Au} \mathrm{XVII}^{\mathrm{e}}$ siècle, l'Eglise réformée de Paris «recueillie à Charenton» ${ }^{6}$, compte 8 à 10000 fidèles (sur 300000 habitants en 1600, 430000 en 1650) et entretient quatre pasteurs et éventuellement quelques proposants (futurs pasteurs). Les pasteurs sont d'abord des prédicateurs, donc des exégètes de la bible et des orateurs; en France, ils doivent

Jacques PAnnier, «La bibliothèque de l'Eglise réformée de Paris, de 1626 à 1664», BSHPF, 1906, p. 40-68. Repris et complété dans: Jacques Pannier, L'Eglise réformée de Paris sous Louis XIII, de 1621 à 1629 environ, Paris, 1932, I, p. 313-322, et II, p. 20-43. Le premier signalement de ce catalogue manuscrit est de Alfred Franklin, Les anciennes bibliothèques de Paris.., Paris, 1873, t. III, p. 444-448.

6 Selon les «articles secrets» de l'édit de Nantes, aucun exercice public du culte réformé n'était autorisé à moins de cinq lieues $(20 \mathrm{~km})$ de Paris, mais des lettres patentes de Henri IV en 1606, autorisent le culte de l'Eglise réformée de Paris à Charenton (Saint-Maurice). 
aussi être des controversistes et des apologètes. Ils ont donc besoin de livres savants de théologie, philosophie, histoire, belles-lettres, voire de droit et de sciences. Plus encore les pasteurs de Charenton, car ceuxci fréquentent la société lettrée et sont aussi des auteurs, imprimant à Charenton ou à Genève leurs sermons, traités, livres de piété ou de controverse. Comme tous les fidèles de l'Eglise de Paris, les pasteurs habitent en ville ou dans les faubourgs, où ils ont leurs propres livres ${ }^{7}$. Mais ils doivent passer beaucoup de temps à Charenton, non seulement pour les servives du dimanche, mais aussi en semaine, à tour de rôle. La bibliothèque sur place leur est d'autant plus nécessaire, pour la lecture ou l'écriture dans les temps morts. Elle peut être utile aussi aux pasteurs occasionnels, qui viennent prêcher à Charenton, par exemple pour les synodes de la province. La bibliothèque est dite «à l'usage public»: non seulement les pasteurs, mais aussi les futurs pasteurs qui n'ont pas encore de bibliothèque personnelle, les anciens du consistoire, les étudiants étrangers en séjour à Paris.

A côté du premier temple de Charenton, édifié en 1607, un bâtiment annexe, construit en 1613, abritait une petite bibliothèque, tout en servant aux séances du consistoire. Le temple fut détruit par des émeutiers en 1621, et les livres volés à cette occasion, mais les murs de l'annexe sont restés debout ${ }^{8}$. Le nouveau temple de Charenton, construit un peu à l'écart du précédent par Salomon de Brosse, fut ouvert en 1624. En novembre 1626, le Synode national de Castres réitéra l'exhortation aux Eglises «les plus riches» à ouvrir des bibliothèques, ciblant en particulier l'Eglise de Paris ${ }^{9}$. De retour à Paris, les délégués de la province d'Ile -de-

Ainsi Jean Mestrezat, pasteur à Charenton de 1615 à 1657, logeait Vieille rue du temple (rive droite); Charles Drelincourt, pasteur à Charenton de 1620 à 1669, rue Princesse (Faubourg Saint-Germain). Les quatre en poste à Charenton au moment de la Révocation, en 1685: Jean Claude habitait rue de Seine, Pierre Allix rue Neuve-Saint-Eustache (rive droite), Jean Daillé fils quartier Sainte-Opportune (rive droite), Jean Mesnard place Maubert. Voir Orentin Douen, La Révocation de l'édit de Nantes à Paris, d'après des documents inédits, Paris, Fischbacher, 1894, t. I, p. 118-122, et Jacques PAnnier, L'Eglise réformée de Paris, op. cit., t. I, p. 316. Aymon., op. cit., II, p. 378, ch. xxvii, art. ii. 
France $^{10}$ firent passer le message et stimulèrent les dons de livres pour constituer un premier fonds de bibliothèque à Charenton, à loger dans la salle du consistoire, dans la maison voisine du temple ${ }^{11}$.

Un catalogue manuscrit des livres est commencé «sur la fin de l'an $1626 »^{12}$, et remplacé huit ans plus tard par un deuxième catalogue manuscrit, intitulé Catalogue des livres de la bibliothèque de l'Eglise réformée de Paris [...] avec les noms de ceux qui les ont donnés [...]. Ce volume relié en veau noir, acheté en $1634^{13}$, portait encore en 1906, sur le plat supérieur, la marque aujourd'hui presque effacée de la Bibliotheca Ecclesiae Reformatae Parisiensis $^{14}$. Le sigle B.E.R.P. surmonte l'emblème de la «vraie religion» dans un médaillon ovale: une femme aux ailes déployées, appuyée sur la croix, foulant aux pieds un squelette (la mort) et tendant à bout de

10 Dont Jean Mestrezat (1592-1657), ministre de Charenton, Isaac d'Huisseau père (v. 1563-1650), ancien de Charenton, et David Blondel (1590-1655), ministre à Houdan-Roussy. Ce même synode de Castres alloue à Blondel une somme de $1000 \mathrm{l}$. pour l'achat de livres, qui lui permettront de rédiger des ouvrages d'histoire, nécessaires pour la défense des réformés dans la controverse, ouvrages dont le synode s'engage à subventionner la publication.

11 O. Douen, op. cit., I, p.l19.

12 Catalogue probablement tenu par Isaac d'Huisseau père, qui a «composé» les registres de l'Eglise (BMS) à partir de 1624 (Jacques PANNIER, L'Eglise réformée de Paris... op. cit., t.I, 1932, p. 290) et était présent au synode de Castres de 1626.

13 «1634... Deux livres blancs, reliez en veau noir. pour escrire les catalogues des Livres de la Bibliothèque et les noms de ceux qui les ont donnez» (Catalogue, f. 10r). On ne sait ce qu'il est advenu du $2^{e}$ livre blanc, ni l'uage pour lequel il était prévu (catalogue Auteurs? ou Matières?). Les f. 1-17 du Catalogue sont d'une même main, du f.17v (un don daté du 2 octobre 1634) jusqu'á la fin des inscriptions (f. 19), d'une autre main. Les f. 20-189 sont blancs.

14 Depuis l'édit de Beaulieu (1576), la mention «Eglise réformée» devait être remplacée dans tous les documents publics par «Eglise prétendue réformée». Les initiales B.E.R.P. permettaient de contourner cette obligation humiliante. 
bras le Livre ouvert ${ }^{15}$. C'était l'ex-libris des livres de la bibliothèque de Charenton, comme en témoignent deux exemplaires aujourd'hui conservés dans des bibliothèques publiques: l'un de ces ex-libris gravé sur la reliure du livre (comme pour le catalogue), l’autre sur papier, collé au contreplat du livre.

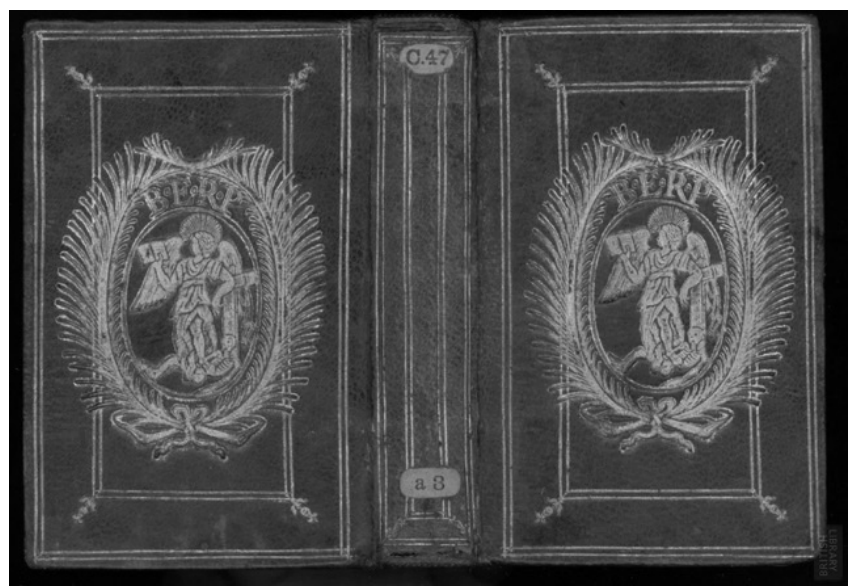

Figure 1. Salvien de Marseille, [...] De vero judicio et providentia Dei ... libri VIII., cura J. A. Brassicani ... editi, [...], Paris, Jerôme de Marnef et Guillaume Cavellat, 1594, in-12 (British Library, C47a3).

15 Sur cet emblème, dont la source est une épigramme latine de Théodore de Bèze (1548), devenue à Genève «Religionis non papisticae sed verae evangelicae pictura» (1559) ou «Portrait de la vraye religion» (1561), et qui est passé en 1580 dans le recueil des Icones de Bèze, voir E. Frossard, BSHPF, 1881, p. 174-182. L'image, associée au poème dès 1560 (Confessione della fede christiana di M. Theodoro Beza.., Genève, Fabio Todesco), est peut-être due au lyonnais Pierre Eskrich (R. Stawarz-LuginbüHL, "Les Emblemata/Emblemes chrestiens (1580/1581) de Théodore de Bèze: un recueil d'emblèmes humaniste et protestant". BHR, LXVII (3), (2005), p. 601). Détachée du poème de Bèze, cette image, en plusieurs variantes, a connu une diffusion exceptionnelle, étant devenue la marque typographique de plusieurs imprimeurs réformés, à Genève, La Rochelle, Charenton, aux XVI et $\mathrm{XVII}^{e}$ siècles. Elle a pris une connotation subversive en 1621 en devenant le sceau de l'assemblée des réformés à La Rochelle, unis dans la résistance à l'oppression; 


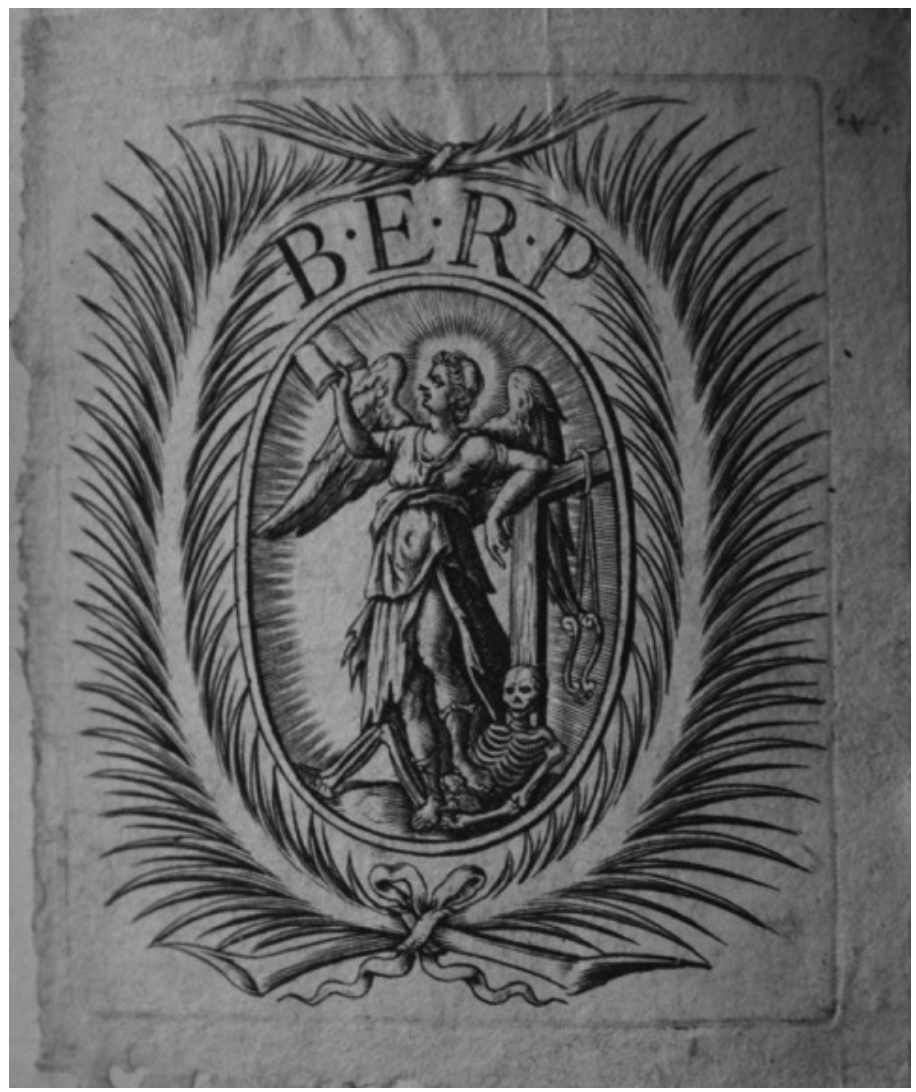

Figure 2. Alexandrea Piccolominı, Della institutione di tutta vita dell'huomo nato nobile, et in citta libera, Venise, G. Bonelli, 1552, in-8 (Venise, BN Marciana, C 068C 204)

Le catalogue manuscrit comporte 336 articles (titres ou séries d'ouvrages d'un auteur), et n'a pas été poursuivi au-delà de 1669 , date des dernières éditions mentionnées.

En 1685 en tout cas, c'est la fin de la bibliothèque, avant même l'édit de Fontainebleau. Un arrêt du Parlement de Paris, du 6 septembre 1685, ordonnait de saisir tous les livres de la R.P.R. portés sur le catalogue des 
livres interdits qu'avait dressé l'archevêque de Paris le $l^{\text {er }}$ septembre (500 articles: titres ou totalité des ouvrages d'un auteur). Aussi le 28 septembre 1685, la police perquisitionne à Paris chez les ministres et anciens, pour saisir ces livres, puis se déplace à Charenton ${ }^{16}$. Là, les commissaires de police constatent l'absence de livres dans le temple et dans la maison du consistoire $^{17}$. Le matin, lorsqu'ils avaient interrogé le pasteur Jean Claude (1619-1687), celui-ci avait indiqué qu'il avait expédié sa bibliothèque, «il y a environ un mois», chez son fils Isaac Claude (1653-1695), pasteur à La Haye $^{18}$. De fait, des lettres de Jean Claude à Isaac évoquent discrètement les balots de livres envoyés à La Haye à la suite de l'arrêt du 6 septembre ${ }^{19}$. Sans doute, ne s'agissait-il pas seulement de sa propre bibliothèque, mais aussi, en même temps, de celle de Charenton ${ }^{20}$. Le 21 octobre, veille de

16 Le cas de la bibliothèque de Charenton n'était pas isolé: bien d'autres bibliothèque consistoriales ont été saisies au même moment (voir Elie BENOIT, Histoire de l'Edit de Nantes..., Delft, 1693-1695, III, l. xxii, p. 829).

17 Voir le procès-verbal des commissaires de La Marre et Lepage, le 28 septembre 1685, «sur les deux heures et demye»: «Sommes ensuite entrez dans une salle basse de l'un des corps de logis joignante ledt temple, que ledt Neveu [concierge du temple»] nous a dit estre le consistoire et lieu d'assemblée, dans laquelle salle ne s'est trouvé aucuns livres. Et nous ayant fait ouverture d'une chambre joignant ladt salle qu'il nous a dit estre la chambre des meditations et le lieu où se retirent les sieurs ministres les jours d'exercice d'avant et après le sermon, avons trouvé en ladte chambre les livres qui ensuivent...», savoir deux Bibles et deux Nouveau Testament (BnF, Manuscrits Français 21740, f. 176).

18 BnF, ibid., f. 173.. Cf. O. Douen, op. cit., II, p. 138 et I, p. 578. - Isaac Claude (1653-1695), à La Haye depuis avril 1682, est nommé pasteur de l'Eglise wallonne de La Haye en juin 1685.

19 Lettres du 14 et du 21 septembre 1685 (Bibl. université de Leyde, B.P.L 292), aimablement communiquées par Helwi Blom (Radboud University, Nimègue), que je remercie pour son aide précieuse dans la recherche de la bibliothèque de Claude.

20 Les lettres de Claude, vagues à dessein, ne permettent pas de l'affirmer. La preuve du transfert de la bibliothèque de Charenton chez Isaac Claude à La Haye vient d'être apportée par des documents découverts aux Archives nationales de La Haye par Helwi Blom, qui les a présentés au colloque «Élie Bouhéreau and the World of Huguenots», Dublin, nov. 2019 (actes à paraître) 
l'enregistrement de l'édit de Fontainebleau, Jean Claude est expulsé du royaume; il se réfugie chez son fils à La Haye, où il meurt en janvier 1687. La bibliothèque de Charenton est restée chez Isaac Claude, à côté de celle de son père, enrichie par ses propres soins.

A la mort d'Isaac Claude, âgé de 42 ans, le 30 juillet 1695, après celle de sa femme, Emilie Briot en 1688, ses enfants orphelins, Jean-Jacques (né en 1684) et Isaac-François (né en 1686), sont confiés à un parent tuteur, David Martin (1639-1721), pasteur réfugié à Utrecht, réviseur de la bible de Genève en français. En novembre 1695, est annoncée la mise en vente aux enchères des «beaux livres» d'Isaac Claude, ancien pasteur à La Haye, avec une grande partie de la bibliothèque de Charenton (maxima pars instructissimae Bibliothecae Reformatae Charentoniensis Ecclesiae). Le catalogue, imprimé par Abraham Troyel, rassemble 2795 articles de toutes sortes, en latin et surtout en français.

C'est peut-être David Martin, pressé de vider la maison des Claude et de récupérer des liquidités pour l'entretien de ses pupilles ${ }^{21}$, qui avait négocié la vente avec Troyel ${ }^{22}$. Avertis par le catalogue, d'anciens fidèles de Charenton, réfugiés aux Provinces-Unies, s'émeuvent, d'autant qu'avant la paix de Ryswick, beaucoup rêvaient encore d'une restauration des Eglises réformées en Farnce. Ces fidèles font retirer de la vente les livres provenant de la bibliothèque de l'Eglise de Charenton ${ }^{23}$. Donc, en principe, en novembre 1695, seuls sont

21 D'où aussi la procédure judiciaire entamée par David Martin en 1698, mentionnée ci-dessous note 26 .

22 Troyel avait l'habitude de ces ventes associant deux ou plusieurs bibliothèques. Voir les remarques des frères Huygens au sujet de la vente de la riche bibliothèque de leur père, Constantyn Huygens (†1687) en 1688, et de son catalogue, dans Christaan Huygens, Euvres complètes, éd. La Haye, M. Nijhoff, t. 9, 1901, lettres des 9 oct., 13 oct., 29 oct. 1687. Les pièces découvertes par Helwi Blom (cf. note 20 ci-dessus) infirment mon hypothèse: c'est la veuve de Jean Claude, Elisabeth de Malécare (†12 février 1696), qui a négocié la vente avec Troyel en octobre 1695.

23 Ernst Ferdinand Kossmann, De boekhandel te 's-Gravenhage tot het eind van de achttiende eeuw, La Haye, 1937, p. 292 et 413. Nouveaux détails dans la communication de Helwi Blom, citée note 20 ci-dessus. 
vendus les livres de Claude. Les livres de Charenton sont transférés chez Théodore de Beringhen (1644-1715), figure de la résistance à la Révocation, et l'une des chevilles ouvrières du comité des réfugiés de Hollande, qui depuis 1693, avec Élie Benoist et Pierre Jurieu, cherchait les voies d'un rétablissement de l'édit de Nantes.

En septembre 1706, la bibliothèque était toujours en dépôt dans la maison Beringhen à La Haye. A cette date, Jean-Jacques Claude ${ }^{24}$, l'un des deux fils du pasteur défunt de La Haye et petit-fils du pasteur de Paris, est reçu proposant par le synode de Deventer, et est demandé par l'Eglise wallonne de La Haye. Celle-ci lui remet en guise d'hommage la bibliothèque de Charenton. En mai 1710, avant d'accepter le poste à La Haye, le jeune homme reçoit l'appel de l'Eglise française de Londres, et le 11 février 1711 il y est installé comme pasteur. Un an plus tard, le 7 mars 1712, il meurt brutalement, âgé de 28 ans $^{25}$.

Le sort de la bibliothèque de l'Eglise de Charenton, restée à La Haye, n'était toujours pas tranché. Le seul héritier, le frère de Jean-Jacques, Isaac-François Claude, vivait à Utrecht. A partir de 1719, il s'installe à Paris, comme Hollandais, pour tenter de défendre ses intérêts, contre des collatéraux avides: ceux-ci s'en étaient pris aux biens laissés par sa mère $(\dagger 1688)$, et venaient de gagner une bataille judiciaire ${ }^{26}$. Soutenu par

24 Voir sa notice biographique dans Jaques George de Chaupepie, Nouveau Dictionnaire historique et critique, Amsterdam, 1750, t.II, p. 97-98.

25 Voir la notice biographique en tête de l'ouvrage posthume de Jean-Jacques Claude, Sermons sur divers textes de l'Ecriture sainte, Amsterdam, 1713, p. xix, xxv. Cf. Auguste Bernus, BSHPF 1895, p. 55.

26 Dès 1698 (après la paix de Ryswick), David Martin avait saisi la Chambre des requêtes du Parlement de Paris, pour défendre les intérêts de ses pupilles dans la succession des biens de leur mère Emilie Briot (copie ms BPF, Fonds Romane Musculus, n¹740). Sur l'affaire, voir les extraits de la correspondance des ambassadeurs de Hollande, dans le Bulletin de la Commission pour l'histoire des Eglises wallonnes, t. 5, 1892, p. 346, 370 s. - Sur le séjour de Isaac-Claude à Paris, voir le registre des «Inhumations au cimetière des protestants étrangers de la porte St Martin, entre 1725 et 1737» (BSHPF, 1902, p. 270-275): 17 février 1727: Isaac-François Claude, hollandais, logé en la maison de la veuve Vauquelin, rue Montmartre déclarant à l'inhumation de la fille du chapelain de l'ambassadeur de Hollande. - 22 septembre 1736: François Claude: 48 ans, secrétaire de S.E. M. Van Hop, ambassadeur de Hollande, décédé rue de Richelieu. 
l'ambassade de Hollande, il finit par avoir gain de cause en septembre 1721. A ce moment, David Martin venait de mourir à Utrecht. Isaac - François Claude est resté à Paris. En octobre 1722, un «choix de la bibliothèque de Charenton» (Bibliotheca selecta carentoniensis), des «livres les plus élégants», est vendu aux enchères publiques à La Haye, cette fois sans protestation. Comme l'indique son catalogue, le libraire Jean Neaulme a couplé cette bibliothèque avec une autre, «anonyme» (Bibliotheca Anonymiana) ${ }^{27}$. L'ensemble des deux bibliothèques mises en vente compte plus de 2500 articles (2876 en comptant l'Appendix, ajout de livres arrivés in extremis), classés par format, en séries numériques continues (les deux bibliothèques sont traitées comme une seule en deux parties). La bibliothèque de Charenton ne compte au total que 521 articles. Avec quatre fois plus de titres, couvrant tous les domaines de la théologie, les lettres, arts et sciences, en latin, français et autres langues, la «bibliothèque anonyme» semble être celle d'un pasteur des Eglises wallonnes, plus spécialement un bibliste. Vu la présence de plusieurs exemplaires de la Bible de David Martin et le lien fait par le vendeur entre la bibliothèque anonyme et celle de Charenton, l'anonyme pourrait être David Martin ( $† 1721)$. Vendue par les héritiers Martin avec les livres de Charenton en guise d'appât, la double bibliothèque a été dispersée en six jours (26-31 octobre). Ainsi s'achève l'histoire de la B.E.R.P.

27 Sur la pratique des catalogues de «bibliothèques anonymes», voir Otto LANKHORST, «Les catalogues du «magasin de l'univers»: intérêt et sauvegarde des catalogues de libraires néerlandais de l'ancien régime», in: Le livre entre le commerce et l'histoire des idées. Les catalogues de libraires (XVe $-\mathrm{XIX}^{e}$ siècle), éd. Annie Charon, Claire Lesage et Eve Netchine, Ecole nationale des Chartes, 2011, p. 14. 
Le CATALOGUE MANUSCRIT (1626-1669)

Catalogue des livres: de la bibliothèque de l'église réformée de Paris avec les noms de ceux qui les ont donnés ou de la libéralité desquels ils ont esté achetés, disposé selon l'ordre des temps que les présens ont esté faicts, in-fol., $19 \mathrm{f.}^{28}$

Ce «catalogue des livres» manuscrit n'est pas à proprement parler un catalogue de bibliothèque, mais un registre des acquisitions par dons ou legs, plus ou moins selon l'ordre des entrées, de la fin de 1626 aux années 1660. Il indique les noms, titres et fonctions de 35 donateurs, et pour les livres, présentés en 333 articles, les noms d'auteurs et des titres sommaires, en français, le format, mais non les éditions. Le catalogue soigné des livres de Charenton établi par le libraire Neaulme en 1722, permet d'identifier précisément une bonne partie de ces livres, et de dédoubler plusieurs articles, ce qui porte le nombre des ouvrages à 354, formant 548 volumes ${ }^{29}$.

Copié en 1634 à partir d'un précédent registre, le catalogue a sans doute été recomposé. En efet, les livres les plus symboliques de l’Eglise réformée se trouvent placés en tête: dans l'ordre, les «Centuries de Magdebourg» de Flacius Illyricus (la grande Histoire ecclésiastique protestante), les oeuvres complètes de Calvin, Luther, Melanchthon, puis saint Jérome,

28 BnF, Manuscrits Français 24480. Le volume fait partie des «Petits fonds français» provenant des saisies révolutionnaires. Sur sa page de titre figure une ancienne cote (818) de la Bibliothèque de l'abbaye Saint-Victor, et un ex-libris de cette bibliothèque daté de 1747. Il est possible que cette grande bibliothèque ait hérité d'épaves de la bibliothèque de Charenton, restées en France, dont ce catalogue manuscrit, seul de son espèce parmi les autres manuscrits de Saint-Victor signalés dans les Petits fonds français. Se référant aux dires de l'abbé Jean Lebeuf, A. FrankLIN (op.cit.) avait supposé que les religieuses des Nouvelles catholiques de Paris, qui avaient hérité en novembre 1685 de la maison consistoriale de Charenton, avaient en même temps hérité d'une partie de la bibliothèque de Charenton, plus tard transférée à SaintVictor, mais les procès-verbaux des perquisitions à Charenton en septembre 1685 contrarient cette hypothèse.

29 En attendant une nouvelle édition du catalogue complet, en préparation, on se reportera à l'édition de Jacques PANnier, L'Eglise réformée de Paris... op. cit., t. II, p. 20-43. 
saint Augustin, saint Chrysostome ${ }^{30}$. Tel qu'il est, ce catalogue permet de reconstituer le fonds primitif de la bibliothèque de Charenton, et le cercle des mécènes qui l'ont constitué, avant 1634 (à partir de 1634, les dons sont presque tous des sommes d'argent ou des legs, non plus des livres choisis pour la bibliothèque). Les premiers donateurs - ils sont vingt- ont offert chacun un livre important (format in-folio, en plusieurs volumes), ou une série d'un même auteur. Ce sont des huguenots huppés: quelques-uns de la noblesse d'épée (Saint-Ravy ${ }^{31}$, Chivray ${ }^{32}$, Harlay $^{33}$, De Clermont ${ }^{34}$, Du Bordage ${ }^{35}$ ), davantage de la noblesse d'Etat:

30 Autre indice d'une recomposition en 1634: au moins un don, placé en $6^{\text {e }}$ position, est antérieur à la constitution de la nouvelle bibliothèque de Charenton en 1626 (ci-dessous note 36).

31 SAint-Ravy: famille de gentilshommes du Languedoc, de la R.P.R. En 1630, un «sieur de Saint-Ravy» est «gentilhomme ordinaire de la chambre du Roy». Vers 1636, Jean Daillé, ministre de l'Eglise de Paris, correspond avec Mme de Saint-Ravy (Jacques PANnier, L'Eglise réformée de Paris... op. cit., t. II, p. 20). En 1658, un «sieur de Saint-Ravy» fréquente l'Eglise de Charenton ([Philippe de Villers], Journal d'un voyage à Paris en 1657-1658, éd. A.P. FAugere, Paris, 1862, p. 435).

32 Henri de Chivré († 1638), marquis de LA BARRe (1634): gouverneur de Jargeau en 1621, lieutenant général en 1634, puis maréchal de camp en 1638.

33 Charles de Harlay (†1636), baron de Dolot: en 1625 capitaine de cavalerie, gentilhomme de la chambre du roi, dédicataire d'un ouvrage de Drelincourt, De la persévérance des saints (1625).

34 Henri de Clermont-Gallerande ( $† 1651)$, marquis de Gallerande: petitfils et fils de gentilshommes huguenots engagés dans les guerres de religion, puis dans les assemblées politiques en 1619, préside le synode provincial d'Anjou à Loudun; en 1626, nommé député général des Eglises réformées, sur proposition du synode national de Castres; démissionnaire en 1644.

35 René de Montbourcher (1578-1647), seigneur Du Bordage, principal seigneur protestant du pays Rennais sous Louis XIII (Jean-Luc Tulot, éd. «Les La Trémoille à Vitré pendant le premier XVII e siècle, I. Correspondance de leurs agents (1606-1624)», jeanluc.tulot.pagesperso-orange.fr). 
conseillers du roi et secrétaires du roi (Arnauld ${ }^{36}$, Lhoste ${ }^{37}$, Marbault $^{38}$, Vallée $^{39}$, Chevalier ${ }^{40}$, Herouard ${ }^{41}$, Juste ${ }^{42}$ ), de la chambre du roi (SaintRavy), ou dans la haute administration des finances (de Netz ${ }^{43}$, Du

36 David ArNauld (1570-1620), frère cadet d'Antoine Arnaud (père de Robert Arnaud d'Andilly); devenu en 1606 conseiller du roi et contrôleur général des «restes» (du Trésor), protégé de Sully, comme son frère Isaac Arnaud (15661617), intendant des finances en 1608. Il faut supposer que la série d' ouvrages - de Saint Augustin- dont il a fait don, sont des rescapés de la première bibliothèque de Charenton, sinistrée en 1621.

37 Hilaire Lhoste fils (1602 - post 1667), sieur de Montfermeil; neveu de David Arnauld; devenu en 1623 conseiller et secrétaire du roi, à la suite de son père.

38 Pierre Marbault (v. 1576-1656), sieur de saint-Laurens: secrétaire de Duplessis-Mornay (†1623), puis conseiller et secrétaire du roi; en 1637, député général des Eglises réformées avec le marquis de Gallerande; démissionnaire en 1644; ancien de l'Eglise de Paris. Voir Céline Houzard, «La communauté protestante de Saint-Germain-des-Prés,1635-1640», BSHPF, 1996, p. 409_ 411.

39 François Vallee (1577-1647), duc de Chenailles: conseiller du roi, présidenttrésorier au bureau des finances de Paris, contrôleur général des guerres et maître d'hôtel du roi.

40 Jean Chevalier: secrétaire de la chambre du roi, fermier général des gabelles de Provence et Dauphiné.

41 Jean Herouard († 1638): conseiller du roi et contrôleur général des fermes de France; cousin du Jean Herouard, médecin du roi; époux de Françoise Le Coq (1598-1638), fille de François Le Coq et de Marie Marbault. Voir Céline HouzARD, op. cit., p. 409-4ll.

42 Christophe Justel (1580-1649): conseiller de Henri IV, puis secrétaire du duc de Bouillon (1610-1623), chargé de former la bibliothèque publique établie auprès de l'Académie protestante de Sedan; après la mort du duc Henri en 1623, au service du duc Frédéric-Maurice.

43 Philippe de Netz (ou de Metz) ( $† 1^{\text {er }}$ décembre 1626): auditeur des comptes, intendant des affaires des La Tremouille à partir de 1615, ancien de l'Eglise de Charenton de 1610 à 1626. 
Temps $^{44}$, Maupeou $\left.^{45}\right)$. Certains ont déjà eu une longue carrière dans le sillage de Henri de Navarre, puis Henri IV (Harlay, De Clermont, Hotman de Villiers $\left.{ }^{46}\right)$. D'autres étaient de l'entourage de Sully, restés en charge sous Louis XIII (David Arnaud, Maupeou, Malleray ${ }^{47}$ ). Plusieurs ont joué un rôle dans les assemblées politiques des réformés jusqu'en 1629 (Malleray, Chivray, Du Matz ${ }^{48}$ et de Clermont, devenus l'un et l'autre députés généraux des Eglises réformées, Marbault, le secrétaire de Du Plessis Mornay). Plusieurs n'habitaient pas Paris (du moins SaintRavy, Malleray, Chivré, Montbourcher, Justel, Martineau $\left.{ }^{49}\right)$, signe d'un intérêt pour la bibliothèque au-delà l'Eglise locale. Deux seulement

44 François Du Temps († avant 1648): trésorier de l'extraordinaire des guerres de Metz, Toul et Verdun; en 1634, avait épousé Marie Marbault, fille de Pierre Marbault (note 38 ci-dessus).

45 Gilles de Maupeou (1553-1641): entré au service de Sully en 1600, tour à tour intendant des finances et contrôleur général des finances (1602-1621); en 1636, dédicataire d'un ouvrage de Pierre Du Moulin; en 1640, converti au catholicisme.

46 Jean Hotman de Villiers (1552-1636): fils aîné de François Hotman, conseiller et maître des requêtes de l'hôtel (1595), diplomate au service de Henri IV. Voir Ferdinand de Schickler, «Hotman de Villiers et son temps», BSHPF, 1868, p. 98, 145, 401, 464 et 513.

47 André Malleray, sieur de Larceau († post 1640): protégé de Sully, fermier de l'abbaye de l'Absie en Gâtine, avocat en parlement à Fontenay-le-Comte, avocat au présidial de Poitiers; ancien du consistoire de Poitiers en 1607; député de la province de Poitou à l'assemblée de Grenoble (1615), à Loudun (1618), à La Rochelle (décembre 1620 et avril 1621), et à la suite pendu en effigie à Poitiers en 1621 .

48 Esaïe Du Matz (1577-1647), sieur de Montmartin: gentilhomme poitevin, avait épousé une petite-fille de Duplessis-Mornay; député général des Eglises réformées de 1622 à 1626.

49 Pierre Martineau ( $\dagger$ entre 1641 et 1644), sieur du Perron: gentilhomme ordinaire des chasses; secrétaire de Charlotte de Montmorency, duchesse d'Angoulème (1628-1636), secrétaire de Gaston d'Orléans (1641-1644?). Voir Idelette Idelette Ardouin, «Ministres et fidèles de l'Eglise réformée de l'Ile-Bouchard, ...», BSHPF, 1990, p. 179-180. Sans rapport - autre que l'homonymie de terres, avec Jacques Davy, sieur du Perron (1556-1618), évêque d'Evreux, dont il offre les oeuvres à la bibliothèque de Charenton. 
étaient des anciens de l'Eglise de Paris (de Netz, Marbault). Une seule femme, une fidèle sans pedegree, $\mathrm{M}^{\text {elle }}$ Le Roy ${ }^{50}$.

Les premiers livres sélectionnés pour fonder la bibliothèque de l'Eglise réformée de Paris, une quarantaine, en 140 volumes, sont des éditions in-folio, dont la moitié du XVI siècle, presque toutes en latin. On trouve d'abord des éditions de sources, les meilleures disponibles, en premier lieu les Opera omnia des réformateurs (éditions de Genève, Bâle, Wittenberg) et celles des grands Pères de l'Eglise (éditions de Paris), ainsi que les séries des conciles anciens (éditions de Cologne et de Rome). Puis les oeuvres des ténors français de la controverse confessionnelle, au début du XVII siècle: d'un côté Du Plessis-Mornay (éditions de Genève, Leyde, Francfort), de l'autre son adversaire, le cardinal du Perron (éditions de Paris, 1622-1623); en dernier lieu le Panstratiae catholicae (ou «guerres de l'Eternel») de Daniel Chamier, tout juste sorti à Genève en 1626. Les éditions savantes de la Bible et des Pères sont aussi au service de la controverse. Non pas les genevoises que devaient avoir tous les pasteurs, mais celles de Plantin à Anvers: la Bible de Louvain corrigée $\left(1565^{51}\right)$, la Polyglotte d'Arias Montanus (15681572) ${ }^{52}$; et de plus profilées de la Contre-Réforme: la Bible glosée de Douai (1617), le Nouveau Testament commenté par le jésuite Salmeron, revu par Bellarmin (Cologne, 1604), et la monumentale «Bibliothèque des Pères» de La Bigne (Paris, 1624). Erasme fait contrepoids avec ses Opera omnia, dans la grande édition Froben de 1540, la seule disponible depuis l'Index de Rome en 1559.

$50 \quad \mathrm{M}^{\text {elle }}$ Le Roy est la dédicataire de la Deuxième décade de sermons (Genève, Jacques Chouet, 1645) de Pierre Du Moulin qui l'avait connue lors de son ministère à Paris, entre 1599 et 1615, et la dit très âgée, pieuse et charitable.

51 Bible latine corrigée sur des manuscrits latins par l'Université de Louvain (Jean Henten), dès 1547, pour se substituer aux éditions de Estienne, mises à l'Index en 1546 ( 1546: le Concile de Trente déclare l'authentícité de la Vulgate et demande une édition aussi pure que possible).

52 Catalogue 1722, n³: «exemplar elegantissimus» (83 l.). Le Synode de Gap en 1603 recommandait l'achat de la Polyglotte d'Anvers pour les bibliothèques des académies (Aymm, op. cit., II, p. 276.). 
Certains dons sont personnalisés. Ainsi les oeuvres de saint Augustin proviennent d'un membre de la famille Arnauld; celles de DuplessisMornay de Marbault, son ancien secrétaire; celles du cardinal Du Perron ont été données par un dénommé «sieur du Perron», jouant sur l'homonymie du nom; les œuvres de François Hotman ont été apportées par son fils Jean Hotman de Villiers; Christophe Justel a offert ses propres œuvres d'historien des codex canoniques; et entre deux conversions, Maupeou a donné les Annales de Baronius (Venise, 1600-1612), réplique catholique aux Centuries de Magdebourg.

Le premier fonds de la bibliothèque de Charenton présente ainsi une large sélection de sources, dans des éditions savantes et en séries d'œuvres complètes, comme instruments de travail des pasteurs.

Les acquisitions à la suite de ce premier fonds, à partir de 1634, représentent 315 ouvrages (environ 400 volumes), provenant de seize donateurs, dont douze par dons d'argent spécifiques, et trois par legs ou dons de livres. Parmi les donateurs de sommes pour les acquisitions, de 44 à $200 \mathrm{~F}$ (ou livres), on retrouve de grands noms de la noblesse huguenote, moins celle d'épée (marquis de Courtomer ${ }^{53}$, comte de La Suze $\left.{ }^{54}\right)$ que d'Etat, avec trois conseillers et secrétaires du roi (Launay ${ }^{55}$,

53 Jean-Antoine Saint-Simon, marquis de Courtomer (v. 1565-1629): épouse en 1595 Marie de Clermont-Gallerande, sœur du marquis de Gallerande, député général des Eglises réformées (ci-dessus note 34); colonel d'un régiment et lieutenant général des troupes françoises en Hollande, tué au

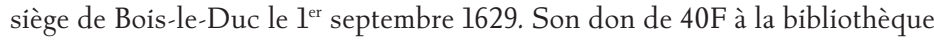
a dû être posthume, fait par sa veuve

54 Louis de Champagne, comte de La Suze (†1636): un des chefs militaires des huguenots pendant les guerres de Rohan, député général des Eglises réformées en 1626, embastillé en 1628, devenu lieutenant général des armées du roi en 1633; époux de Charlotte de La Rochefoucault (†1637), sœur de François de La Rochefoucault (ci-dessous note 57).

55 Pierre de Launay (1573-1661): ancien de l'Eglise réformée de Paris pendant plus de quarante ans; passionné d'exégèse, auteur de plusieurs commentaires bibliques, dont deux, publiés en 1619 et 1624, qu'il donne à la bibliothèque de Charenton. 
Rambouillet ${ }^{56}$, La Rochefoucauld ${ }^{57}$ ). Ajoutons-y le secrétaire de Gaston d'Orléans, qui en 1647 lègue 200 écus à l'Eglise de Paris pour des achats de livres ${ }^{58}$. Mais les donateurs sont aussi des étrangers fréquentant le temple de Charenton: l'ambassadeur des Provinces-Unies à Paris ${ }^{59}$, et plusieurs jeunes Anglais en séjour à Paris, en 1634-163560.

Avec ces dons en argent, le consistoire a acheté 164 ouvrages (en 225 volumes), tous en latin, ou grec ou latin-grec (et 2 en hébreu), la plupart en format in-folio. Près de la moitié sont des impressions parisiennes (75) et une douzaine lyonnaises, pour à peine une quarantaine d'impressions de Genève et de Suisse (19 de Genève, 12 de Bâle, 7 de Zurich). Les autres impressions se dispersent entre Francfort (7), Londres (6), Amsterdam (2), Cologne (6), Anvers (5), Rome (3), Louvain (2), Leyde (2)... Les achats à l'étranger se font en bloc, par ville, pour limiter les frais de port. Les deux -tiers des acquisitions (120) sont des éditions du XVII e siècle (pour la plupart, des années 1600-1635, 8 seulement postérieures à 1650), mais

56 Nicolas de Rambouillet (1576-1664): riche financier, ancien de l'Eglise réformée de Paris. Voir Jacques Pannier, L'Eglise réformée de Paris..., op. cit., t. I, p. 398-401.

57 François de La Rochefoucauld-Roye (1603-1680), comte de Roucy, épouse en 1627 Julienne-Catherine de La Tour d'Auvergne ( $† 1638)$, sœur de Turenne; beau-frère de La Suze (ci-dessus note 54). Voir E. et E. HAAG, La France protestante, t. VI, 1856, p.354.

58 Calixte Musnier (†1647). Son legs a été difficilement exécuté par les héritiers, en deux temps, en 1654, puis 1664 (voir les mentions dans le Catalogue manuscrit).

59 Gideon van Boetzelaer, baron de Langerack et de Asperen (1569-1634): ambassadeur à Paris depuis 1614, jusqu’à sa mort en janvier 1634, suivait de près les affaires des Eglises réformées; en 1625, avait épousé Elisabeth de Clermont-Gallerande, sœur du marquis de Gallerande, député général des Eglises (voir ci-dessus note 34). Le don à la bibliothèque a sans doute été posthume, fait par sa veuve.

60 En juin 1634, Francis Russel, comte de Bedford (1593-1641), devenu en 1640 le chef du parti populaire dans le Long Parlement. En 1635, trois jeunes gens accomplissant leur tour sur le continent: James StUART, duc de Lennox (1612-1655), qui fréquentait Saumur; Thomas Craven (1618-1636), mort de la peste à Charenton;William Cavendish de Hardwick (1617-1684), comte de Devonshire, chaperonné en France par son précepteur Thomas Hobbes. 
un tiers sont du $\mathrm{XVI}^{\mathrm{e}}$ siècle et 4 sont des incunables, achetés donc chez des libraires d'occasion. Les prix d'achat des livres sont indiqués pour les 26 du don du comte de Bedford, en juin 1634.

Dans les acquisitions, les bibles et les théologiens «modernes» ne sont pas en position dominante. C'est sans doute que ces livres faisaient le fonds principal des bibliothèques personnelles des pasteurs. Deux bibles seulement figurent parmi les acquisitions de la bibliothèque depuis 1634: la nouvelle Bible grecque de Sixte V (Paris, 1628), et une édition «en vieux françois et lettres gothiques» (Paris, 1520) ${ }^{61}$. Le Talmud en hébreu (Amsterdam, 1644-1647) a été acquis en 1654, en même temps qu'un dictionnaire arabe-latin (Leyde, $1653^{62}$ ). Pour les théologiens des $\mathrm{XVI}^{\mathrm{e}}$ et XVII ${ }^{\mathrm{e}}$ siècles, une quarantaine d'ouvrages: du côté protestant, des œuvres d'exégètes (tels Oecolampade, Zanchi, Jean Mercier), de dogmaticiens controversistes (Johann Alstedt; Actes du synode de Dordrecht, 1620), plusieurs histoires des pratiques religieuses (du zurichois Rudolf Hospinian); du côté catholique, quelques ouvrages d'exégèse (Jean de Lorin), de controverse (Thomas Stapleton), d'histoire ecclésiastique (Hébert Rosweyd) et plusieurs collections de droit canonique.

Les acquisitions ont surtout renforcé le fonds patristique: au moins 45 éditions, dont 28 Opera omnia, en majorité des Pères grecs, et elles ont permis de constituer un fonds important de textes du Moyen-Age: une quarantaine d'éditions comprenant les œuvres complètes de théologiens tels Hugues de Saint-Victor, Bonaventure, Ockam, une quinzaine de chroniques ecclésiastiques, et des collections de droit canonique. Elles ont aussi permis une extension du fonds de la bibliothèque à l'antiquité gréco-latine, et plus largement à l'histoire et à la philologie: au total, une quarantaine d'ouvrages. Ainsi les histoires de Tite Live et de Thucydide, La Mer des histoires, Res hungaricae d'Antonio Bonfini (1543); les Opera

61

Décrite ainsi dans le Catalogue Neaulme de 1722.

62 Ce dictionnaire «que nous achettasmes autre fois pour la Bibliothèque de nôtre Eglise nous en cousta 25 [livres]», écrivait le pasteur Jean Daillé fils à Louis Tronchin, son collègue de Genève, le ${ }^{\text {er }}$ mars 1661 («Correspondance de Jean Daillé fils», éd. Jean-Luc TuLot, jeanluc.tulot.pagesperso-orange.fr). 
omnia d'Aristote, Platon, Cicéron, Sénèque, Plutarque; un choix de littérature antique, grecque et latine, dans des éditions de Isaac Casaubon ou autres; le Thesaurus de la langue grecque d'Henri Estienne (1572), des œuvres de Guillaume Budé; des dictionnaires; en dernier lieu des «ana» (recueil de «bons mots, pensées judicieuses, observations curieuses»: Scaligerana, de 1668, Thuana, de 1669).

A côté de ces acquisitions volontaires, le catalogue fait apparaître un lot considérable constitué par le legs d'un fidèle, Ambroise Hubert, «bourgeois de Paris», fort riche grâce à Henri IV, mort le 26 juillet $1634^{63}$. Il lègue à l'Eglise de Paris ses 136 livres de théologie et de piété, qui sont presque tous (à 95\%) en français et dans de petits formats: éditions de Genève, Sedan, La Rochelle, Charenton, Saumur, Leyde...; pour moitié du XVII ${ }^{e}$ siècle, dont une trentaine des années 1625-1631. Des sermons, des commentaires bibliques, des ouvrages de controverse, des recueils de prières, des manuels de consolation, de catéchisme, de morale. Les auteurs: Calvin (12 ouvrages, dont 9 in-folio), Bèze (4), Duplessis-Mornay (7), les pasteurs de Charenton (23, dont 4 Durant, 13 Du Moulin, 2 Mestrezat, 3 Drelincourt, 1 Le Faucheur), deux pasteurs anglais en traduction française (Joseph Hall et Lewis Bayly). La Bible en français - trois bibles de Genève, un Nouveau Testament de La Rochelle - et en éditions savantes - la Biblia de Robert Estienne (1545), et celle de Bèze et Tremellius (1602), une concordance biblique en latin, un index biblique, et un Nouveau Testament bilingue grec-latin, avec la version d’Arias Montanus (Genève, 1627). Après ce legs d'Ambroise Hubert, le catalogue ne recense que de rares dons de livres ${ }^{64}$.

63 Voir, en date du 3 mai 1601, les lettres patentes du roi Henri IV portant bail de la douane de Vienne à Ambroise Hubert, bourgeois de Paris, pour 2 ans et 8 mois, au prix de 16000 écus par an (Arch. dép. Isère, Inv.sommaire, 1884, p. 146).

64 Sans compter Pierre de Launay, donateur de deux de ses œuvres outre d'une somme d'argent, ce sont «M. de Jousse» pour un Duplessis-Mornay; Guy de Morogues, gendre d'Hilaire de Lhoste, secrétaire du roi, pour deux livres de Nicolas Vignier; et en 1654, Jacques SarRazIN (1594-1663), natif de Genève, un des médecins de Louis XIII, pour 9 livres variés de sa bibliothèque. 
Si l'on met à part le legs Hubert, qui apporte une dimension proprement pastorale à la bibliothèque, on peut se faire une idée précise de la politique d'acquisition bibliographique de l'Eglise réformée de Paris. La B.E.R.P., quoique sans manuscrit ${ }^{65}$, se veut une bibliothèque savante, rassemblant sous quelque 200 articles toutes les grandes collections de sources, en format in-folio: la bible et les œuvres des auteurs passés et récents dans des éditions «scientifiques» et complètes, codes, sommes, recueils d'histoires. Sans surprise, la théologie, incluant les Pères et l'histoire ecclésiastique, occupe près des trois-quarts du fonds, le quart restant étant constitué des auteurs de l'Antiquité, de chroniqueurs et d'humanistes. L'orientation générale est l'exégèse biblique, la controverse, les «humanités». On note la part importante des ouvrages en grec ou bilingues latin-grec - Pères de l'Eglise et théologiens byzantins- et auteurs de l'Antiquité grecque: environ 22\% du fonds de livres. D'après ce catalogue, la bibliothèque de Charenton se rapproche du fonds d'une bibliothèque d'académie, telle celle de Saumur, constituée à la même époque, mais d'une autre ampleur (1441 titres) ${ }^{66}$. Tous ces ouvrages sont des ressources pour les pasteurs de la capitale, en ligne de mire dans leur rôle d'exégètes bibliques, et en situation de controverse.

Cependant, le fonds de livres dans le catalogue manuscrit - 354 titresparaît quantitativement faible, comparé à la bibliothèque d'une Eglise modeste telle celle d'Issoudun en 167967. Bizarrement, le registre est interrompu en 1669, alors qu'il restait 169 feuillets blancs déjà préparés, foliotés. Il est probable que le catalogue manuscrit ne recouvre pas la totalité du fonds de la bibliothèque de l'Eglise de Charenton. Permettra de le confirmer le rapprochement avec le catalogue des livres vendus

65 Exception: un manuscrit donné par Pierre Marbault en 1628, d'après le Catalogue 1722, $4^{\circ} \mathrm{n}^{\circ} 92$ : Epitome Philosophiae moralis et institutionum physicarum (cf Catalogue $16954^{\circ} \mathrm{n}^{\circ} 684$ ).

66 Voir Clotilde Perigault: La bibliothèque de l'académie protestante de Saumur en 1685: transcription de l'inventaire et identification bibliographique, Mémoire, Tours, Centre d'études supérieures de la Renaissance, 2004.

67 Sur cette bibliothèque de 532 ouvrages, formant 601 volumes, dont $62 \%$ en latin, 27\% en français, voir Yves GuenEAu, «L'inventaire de la bibliothèque de l'Eglise réformée d'Issoudun au XVII e siècle», BSHPF, t. 131, 1985, p. 71-103. 
à La Haye en 1722, présenté comme une «sélection» de livres de la bibliothèque de Charenton parvenus à La Haye en 1685, puis dans une certaine mesure avec le catalogue de vente de 1695, moins aisément exploitable.

Les catalogues de vente de La Haye, 1722 et 1695

Bibliotheca selecta Carentoniensis, Sive Catalogus elegantissimorum librorum inter quos excellunt Theologi, Patres, Historiae ecclesiasticae, et Prophanae scriptores, Litteratores, aliique Miscellanei optimae notae, \& raro occurrentes. Olim usui publico dicati in Ecclesia R.Carentoniensi. His accessit Bibliotheca Anonymiana excellentissimis cujuscunque facultatis, Artis \& Scientiae, in vario linguarum genere libris referta.

Vente La Haye, Joannis Neaulme,

$$
\text { [26-31 oct.] 1722,[ii] p.1-32- [ii] p. 33-124, [1 pl] }{ }^{68} \text {. }
$$

Comme indiqué au titre, la Bibliotheca selecta Carentoniensis mise en vente est constituée des plus beaux livres des «theologiens, des Pères de l'Eglise, des histoires ecclesiastiques et prophanes, des belles-lettres et mélanges». Le classement des 521 articles (551 titres d'ouvrages) est seulement par format: 181 in-folio, 196 in $-4^{\circ}, 130$ in- $8^{\circ}, 14$ in- 12 . Pour les formats $4^{\circ}, 8^{\circ}$ et $12^{\circ}$, se présentent des sous-sections de livres en italien, en tout 87 livres (dont 62 in- $4^{\circ}$ ), donnés ou légués à l'Eglise réformée de Paris, par un collectionneur italien de livres de littérature et de philosophie, pour la plupart du XVI ${ }^{e}$ siècle, imprimés à Venise ${ }^{69}$. Hormis

68 Ce catalogue, mentionné dans le catalogue de la bibliothèque de Nuremberg en 1774, est conservé dans plusieurs bibliothèques publiques, dont la BnF.

69 Le don ou le legs postérieur à 1646, date du plus récent des 87 livres italiens (dont 47 imprimés à Venise). La Présence d'un traité en défense de Galilée fait penser que le lot de livres pourrait provenir du savant lettré Elie Diodati, né à Genève, fils d'un réfugié de Lucques, installé à Paris depuis 1602, inhumé à Charenton le 17 déc. 1661. L'un de ces livres, A. Piccolomini, Della institutione di tutta vita...,Venise, 1552, portant l'ex-libris de la B.E.R.P. avec l'emblème de la «vraie religion» (fig. 2), porte aussi au frontispice, un ex-libris manuscrit «Mauricius Beguynus» (la famille Béguin fait partie du patriciat de Genève au $\mathrm{XVI}^{\mathrm{e}}$ siècle). 
les 87 livres en italien, et 11 en français, toute la bibliothèque mise en vente est en latin (ou latin-grec).

On ne retrouve que 134 ouvrages du catalogue manuscrit de la bibliothèque (dont 119 in-folio). Manquent en particulier presque tous les livres du legs Hubert, la plupart en français, de petits formats, mais aussi une cinquantaine des acquisitions en format in-folio, dont les éditions de Calvin, Melanchthon, Bucer, Zanchi, Cassander, de même les Duplessis-Mornay dans tous les formats. S'agissant des livres de la «R.P.R», sur la liste des livres interdits d'août 1685, il se peut que le pasteur Claude les ait séparés des livres qu'il envoyait en septembre à La Haye et les ait expédiés ailleurs ${ }^{70}$. Mais il manque aussi la Bible latine de Louvain-Plantin, Jean Damascène, Lactance, les Histoires de Pline, des dictionnaires. Des éditions savantes in-folio, du XVI ${ }^{\mathrm{e}}$ siècle, ont peut-être été remplacées par de plus récentes. Ou bien les livres ont été perdus ou volés dans les transports, ou empruntés à La Haye, ou encore le libraire Neaulme les a jugés sans intérêt pour la vente ${ }^{71}$.

Quant aux quelque 350 livres du catalogue de 1722 ne figurant pas au catalogue manuscrit, ce sont des ouvrages du XVI ${ }^{e}$ comme du XVII siècle, mais aucun n'est postérieur à 1669 (date de la Bible en français de Desmarest, d'Amsterdam, en 2 volumes in-folio). La grande majorité de ces ouvrages sont dans les formats in $-4^{\circ}$, et surtout in- $8^{\circ}$ et in- 12 . Mis à part la collection italienne et la dizaine de titres en français, les nouveaux livres sont en latin. On y trouve peu de théologiens «modernes» et très peu de protestants, parmi lesquels un Moyse Amyraut, de Saumur, à côté d'un anti-arminien hollandais, Henri a Dienst. Peu de philosophes «modernes», sinon Descartes (une seule édition, de 1644), Gassendi,

$70 \quad$ Plusieurs des titres des ouvrages manquant dans le catalogue de 1722 sont dans le procès-verbal de saisie des livres des libraires de Charenton (BnF, Manuscrits Français 7050, f. 87-90). Mais on ne voit pas pourquoi ces libraires auraient récupéré des livres de la bibliothèque, interdits depuis août 1685, voués à la destruction.

71 Dans le catalogue de la bibliothèque «Anonymiana» de 1722, Neaulme indique à la fin de l' «Appendix»: «un paquet de 5 in-folio divers» et «un paquet de 9 in-quarto divers». 
Pierre de la Ramée, Joachim Camerarius.... Toujours des Pères de l'Eglise - surtout Grecs- et leurs commentateurs, ainsi Saracenica sive moametica secta, grec-latin, édité par Frédéric Sylburg (Heidelberg, 1595). A noter aussi Maïmonide dans l'édition latine de Buxtorf (Bâle, 1629). Cependant, la grande majorité de ces livres sont de littérature et philosophie de l'Antiquité, d'histoire, de géographie, de médecine (30 ouvrages), de sciences (entre autres plusieurs Jérôme Cardan).

Vu leurs dates, au plus tard de 1669, et leur nombre, il est peu probable que tous ces livres aient été acquis après 1669, moment des dernières acquisitions dans le catalogue manuscrit (même si, au XVII e siècle, les bibliothèques achètent volontiers des ouvrages anciens ${ }^{72}$ ). Le catalogue manuscrit n'a donc pas enregistré toutes les acquisitions jusqu'en 1669, mais seulement les dons de donateurs identifiés.

Le catalogue de vente de 1722 est lui-même affiché comme sélectif: «Bibliotheca selecta carentoniensis». De fait, on peut noter au moins un livre qui a fait partie de la bibliothèque de Charenton, d'après l'ex-libris, et n'est ni dans le catalogue de 1722 ni dans le catalogue manuscrit ${ }^{73}$. On relève aussi des traces d'acquisitions de la bibliothèque, d'ouvrages absents du catalogue, dans la correspondance de Jean Daillé fils, pasteur à Charenton de 1658 à $1685^{74}$; où l'on voit de surcroît l'intérêt des pasteurs de

72 Voir plusieurs lettres de Jean Daillé fils à Louis Tronchin (Correspondance de Jean Daillé fils, éd. Jean-Luc Tulot, op.cit.).

73 Ex-libris B.E.R.P. gravé sur la reliure (voir fig. 1): SALvien de Marseille: D. Salviani Masyliensis Episcopi de vero judicio et providentia Dei... libri VIII., cura J. A. Brassicani... editi, ac eruditis scholiis illustrati. Paris, Jerôme de Marnef et Guillaume Cavellat, 1594, in-12 (exemplaire à la British Library). L'autre exemplaire avec ex-libris B.E.R.P. (cité ci-dessus note 69, cf fig. 2) est, lui, d'une édition figurant dans le catalogue de 1722. Les ex-libris manuscrits «à l'Eglise de Paris» sur quelques ouvrages de la Bpf, attribués à la bibliothèque de Charenton par Jacques Pannier (BSHPF, 84 (1935), p. 54l) et d'autres, indiquent en réalité la provenance de la bibliothèque du chapitre Notre-Dame de Paris.

Ainsi une lettre du 3 septembre 1664 de Jean Daillé fils à Louis Tronchin: «'attendray de vos nouvelles pour le Diogène de M. Ménage [Diogène Laerce, De vitis..., éd. critique annotée par Gilles Ménage, Londres, 1664], en cas que vous en veuilliez faire la dépense pour vôtre bibliothèque publique; car nous l'avons fait pour la nôtre...» (Correspondance de Jean Daillé fils, éd. Jean-Luc Tulot, op.cit.). 
Charenton pour les nouveautés bibliographiques (théologie, spécialement janséniste, sciences bibliques, lettres) et pour les achats de livres. Il serait aussi étonnant que ces pasteurs n'aient pas donné à la bibliothèque leurs propres œuvres, qui figuraient en nombre sur les étals des libraires de Charenton $^{75}$. Or le catalogue de 1722 ne mentionne ni Drelincourt ni Daillé ni Claude, ni leurs adversaires catholiques et jansénistes. Bref, le catalogue de vente de 1722 ne donne qu'un petit aperçu du fonds de livres, sans doute le plus vendable.

Reste encore à interroger un autre témoin du fonds de livres de Charenton: le catalogue de vente présenté par Abraham Troyel en 1695, qui rassemble des livres de Charenton et ceux des Claude père et fils.

Catalogus variorum, insignium, nitidissimorum ac perpulchre compactorum, in omni Facultate \& linguâ Librorum, Praesertim Theologicorum \& Miscellaneorum celeberrimi \& doctissimi viri Isaaci Claude, dum viveret Ecclesiae Hagiensis Gallo-Belgicae Pastoris vigilantissimi \& fidelissimi, quibus addita est maxima pars instructissimae Bibliothecae Reformatae Charentoniensis Ecclesiae.

Vente La Haye, Abraham Troyel, [21] novembre 1695, ii-50 p. ${ }^{76}$

Ce catalogue annonce la vente des livres du pasteur Isaac Claude, mort quelques mois plus tôt, auxquels a été ajoutée la plus grande partie («maxima pars») de la bibliothèque de l’Eglise réformée de Charenton. Les livres venant de Charenton ont finalement été retirés de la vente (d'où la nouvelle vente de 1722). Cependant, la distinction a dû être malaisée, car sur son catalogue le libraire n'a pas séparé cette bibliothèque de celle d'Isaac Claude, en partie héritée de son père Jean Claude. Dans la maison Claude à La Haye, les deux bibliothèques devaient cohabiter, et vu le désordre des titres dans le catalogue, la mise en caisses a dû se faire dans la précipitation.

Voir le P.V. de saisie des «livres trouvez chez les libraires tant à Paris qu’à Charenton», 28-29 septembre 1685 (BnF Manuscrits Français 7050, f. 87-90).

Exemplaires conservés à la Bibliothèque nationale de St Petersbourg et à l'UB d'Augsbourg. Je remercie vivement Frédéric Barbier et Otto Lankhorst de m'avoir permis la consultation de ces exemplaires. 
Au total: 2795 articles, dont 506 in-folio, 737 in $-4^{\circ}, 842$ in $-8^{\circ}, 710$ in- 12 . A la différence de la bibliothèque en vente en 1722 , ici seulement $42 \%$ des articles sont en latin, tandis que $47 \%$ sont en français, $4 \%$ en anglais, un peu moins de $2 \%$ en italien, de même en flamand. Dans les petits formats, les ouvrages en français sont nettement majoritaires: $58 \%$ dans les in $-8^{\circ}$ et $80 \%$ dans les in- 12 .

Sur les 2795 articles, 373 seulement se retrouvent dans le catalogue de 1722 ou dans le catalogue manuscrit (donc 148 articles du catalogue de 1722 ont été négligés en 1695, parmi lesquels 34 des 87 livres italiens): 186 in-folio, 136 in $-4^{\circ}, 47 \mathrm{in}-8^{\circ}, 4 \mathrm{in}-12$. On les trouve placés en blocs compacts à la fin des in-folio, et à la fin des $4^{\circ}$, plus un petit paquet dans les $8^{\circ}$, et plusieurs dizaines disséminés dans les différents formats. On peut supposer que des livres présents au sein de ces blocs, quoique absents du catalogue de 1722 (une cinquantaine), faisaient bien partie de la bibliothèque de Charenton. Ajoutons-y encore quelques éditions du catalogue de 1695 qui font partie de la vente de la bibliothèque «Anonymiana» de 1722, venue de David Martin, et par lui des Claude: a priori, la bibliothèque des Claude ayant été vendue en 1695, il s'agirait de livres de Charenton (ainsi l'Histoire universelle d'Agrippa d'Aubigné, édition de 1616, en deux exemplaires).

En dehors des ouvrages présents ou réputés présents dans le double catalogue de 1722 ou dans le catalogue manuscrit, comment identifier les livres de Charenton dans le catalogue de 1695? On peut en tout cas sortir les ouvrages postérieurs à 1685, voire 1684: à tout le moins une bonne centaine entre 1685 et 1695, pour les formats in-fol. et in$4^{\circ}$ (mais il faudrait identifier les dates d'édition des petits formats, non mentionnées dans le catalogue de Troyel). De même les ouvrages en langue flamande, acquisitions d'Isaac Claude: une cinquantaine. On peut aussi soustraire d'office 110 ouvrages notés comme vendus sur un exemplaire du catalogue conservé à St-Petersbourg, avec les prix d'adjudication ${ }^{77}$. en marge, ces marques ne témoignant pas d'acquisitions. 
Pour le reste, c'est-à-dire la grande masse des livres du catalogue, il est difficile de faire le départ entre les bibliothèques de Charenton et celle des Claude. Ce fonds de quelque 2000 livres tranche avec la sélection de 1722, par la présence massive du français, l'ouverture chronologique des éditions, la variété des sujets. La plus grande place est occupée par la théologie et les sciences bibliques - exégèse et histoire (en latin), la controverse et la pastorale (en français), dans des éditions d'Amsterdam, Leyde, Heidelberg, Londres, Cologne: la Clavis de Flacius Illyricus, Gwalther, Bèze, David Blondel, Samuel Bochart, André Rivet, les pasteurs de Charenton au complet, Louis Cappel, Gomar, de nouvelles éditions de Calvin, des calvinistes orthodoxes et des arminiens, des sociniens et des antisociniens, et du côté catholique, Bellarmin. Certains ouvrages sont assez récents, des années 1670-1685: Jurieu et Bayle, Maimbourg, Nicole, Bossuet, Richard Simon. L'histoire, la philosophie - dont les oeuvres complètes de Descartes (Amsterdam, 1677), Grotius, Hobbes, Locke - la médecine, la géographie, la physique et l'histoire naturelle, présentes dans le catalogue de 1722, sont une composante importante du fonds de 1695. Les récits de voyage et les mémoires ont pu aussi bien être chez les Claude quà Charenton. En revanche, il faut rendre à la bibliothèque personnelle d'Isaac Claude les cartes et atlas, les livres de jardinage (une douzaine) et les «pièces galantes» (une trentaine d'articles couvrant chacun plusieurs titres ${ }^{78}$ ). Il n'empêche que le catalogue de Troyel ne permet pas d'établir avec sûreté le fonds propre de la bibliothèque de Charenton.

78 Après l'édition des œuvres posthumes de son père (1688-1689), le pasteur Isaac Claude a publié (anonymement) une «nouvelle galante»: Le comte de Soissons et le card. Richelieu rivaux de Madame la duchesse d'Elboeuf, Nouvelle galante, Cologne, Pierre Marteau, 1690. Sur le genre de la nouvelle galante, et ses connexions avec la nouvelle historique, voir René Godenne, Histoire de la nouvelle française aux $17^{e}$ et $18^{e}$ siècles, Genève, Droz, 1970, p. 80-85. 
Avec deux catalogues très partiels, et un troisième plus développé mais sans contours nets, l'entreprise de reconstitution du fonds de la bibliothèque de Charenton au XVII ${ }^{e}$ siècle s'avère impossible. Cette enquête décevante peut cependant suggérer d'autres études de cas, en étendant le champ aux bibliothèques académiques et pastorales, et en prenant en compte des sources plus variées que les seuls inventaires ou catalogues de bibliothèques, en particulier les correspondances des pasteurs et les catalogues de libraires. De là, en perspective, une reconstruction des lectures des pasteurs - à la fois lecteurs et auteursdans le contexte particulier de la minorité réformée en France: leurs informations bibliographiques, leur approvisionnement en livres, leurs usages des livres dans leurs propres livres. 
LES BIBLIOTHÈQUES ET L'ÉCONOMIE DES CONNAISSANCES Bibliotheken und die ÖKonomie des Wissens

$$
145^{\circ}-185^{\circ}
$$

Colloque international - Internationale Tagung 9-13 avril/April 2019 Sárospatak (Hongrie/Ungarn)

Édité par

Frédéric Barbier, István Monok et Andrea Seidler 


\section{L'Europe en réseaux}

Contribution à l'histoire de la culture écrite 1650-1918

Vernetztes Europa

Beiträge zur Kulturgeschichte des Buchwesens 1650-1918

Édité par

Frédéric Barbier, Marie-Elisabeth Ducreux, Matthias Middell, István Monok, Éva Ringh, Martin Svatoš

Volume VIII

École pratique des hautes études, Paris

École des hautes études en sciences sociales, Paris

Centre des hautes études, Leipzig

Bibliothèque nationale Széchényi, Budapest

Bibliothèque et centre d'information de l'Académie hongroise des sciences, Budapest 
LeS BIBLIOTHÈQUeS ET L'ÉCONOMIE DES CONNAISSANCES BibliotheKen und die ÖKonomie des Wissens

$$
145^{\circ}-185^{\circ}
$$

Colloque international - Internationale Tagung 9-13 avril/April 2019 Sárospatak (Hongrie/Ungarn)

Édité par

Frédéric Barbier, István Monok et Andrea Seidler

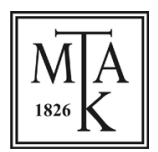

Magyar Tudományos Akadémia Könyvtár és Információs Központ Budapest 2020 
Mise en page

Viktória Vas

ISBN 978-963-7451-57-7

DOI 10.36820/SAROSPATAK.2020 
Préface

István MoNOK

Bibliothecae mutantur - Quare, quemadmodum et quid attinet?

Transformations de la composition thématique

des bibliothèques du Royaume de Hongrie aux $\mathrm{XV}^{\mathrm{E}}-\mathrm{XVI} \mathrm{I}^{\mathrm{e}}$ siècles....11

Marianne CARbonnier-Burkard

Les bibliothèques des Églises réformées françaises au XVII siècle.... 30

Max Engammare

De la bibliothèque de l'Académie de Calvin (1570) a la bibliothèque de l'Académie de Bèze (1612) à travers leur catalogue: Continuités et ruptures jusqu'au troisième catalogue de 1620........... 57

Róbert OLÁ́

Obsolescent Reformed Libraries in the seventeenth and eighteenth Century Carpathian Basin

Ádám Hegri

Moderner Zeitgeist - veraltete Lesestoffe. Bibliotheken reformierten Pfarrer um die Wende des 18. zum

19. Jahrhunderts im Königreich Ungarn

Petr MAšEK

Zierotin Library in Velké Losiny in Sixteenth and Seventeenth century. 136

Detlef HABERLAND

Schlesische Bibliotheken Zeichen der intellektuellen Vielfalt einer zentralen Bildungsregion in Europa 146

Thomas WaLLNIG

Sebastian Tengnagel und Johann Seyfried - Österreichische

Geschichtsschreibung zwischen Späthumanismus und

Gegenreformation 
Elisabeth EngL-Ursula RautenBerg

Christoph Jacob Trew - Bibliothek und Sammeln in der Gelehrtengemeinschaft der ersten Hälfte des 18. Jahrhunderts.

Helwi BLOM

Philosophie ou Commerce?

L'évolution des systèmes de classement bibliographique dans les catalogues de bibliothèques privées publies en France au XVIII ${ }^{\mathrm{e}}$ siècle.

Maria Luisa López-VIDrIero Abelló

Les meubles de la connaissance: façons de devenir sage à prix fixe.

Frédéric BARbier

Distinction, récréation, identité: la trajectoire des « romans» en France sous d'Ancien Régime.

\section{Andrea SeIdler}

Die praktische Bedeutung ungarischer Sammlungen und Bibliotheken für führende Gelehrte des Königreichs Ungarn im späten 18. Jahrhundert am Beispiel des Jesuiten Georg Pray (1723-1801).

\section{Olga Granasztói}

Se divertir: les enseignements de la bibliothèque d'une femme aristocrate hongroise à la fin du XVIII siècle

Christophe Didier

La naissance du théâtre „des boulevards”, ou Comment la banlieue entre en bibliothèque (1780-1830).

\section{Andrea De Pasquale}

La nascita delle riserve di libri antichi in Italia

Index des noms de personne et de lieu..... 